\title{
FORTIFICACIONES FRANCESAS EN EL CARIBE FRENTE A LOS ATAQUES DE LA GUERRA DE LOS SIETE AÑOS ${ }^{1}$
}

\author{
Pedro Luengo \\ Universidad de Sevilla \\ pedroluengo@us.es \\ Ignacio J. López Hernández \\ Universidad de Sevilla \\ ilopez7@us.es
}

\begin{abstract}
Resumen: Los ataques a posiciones francesas en el Caribe durante la Guerra de los Siete Años pueden considerarse un experimento previo al de La Habana, probablemente la victoria más significativa del conflicto en la zona. Para llegar a esta conclusión, este artículo analiza el estado de las construcciones defensivas en Haití, Guadalupe, Martinica, Dominica y Santa Lucia. Para ello se han consultado proyectos de fortificación depositados en diferentes archivos, además de los restos arquitectónicos aún conservados. Posteriormente se abordan los distintos ataques británicos desarrollados entre 1759 y 1762 a partir de fuentes contemporáneas, valorando la relevancia de las fortificaciones en el desenlace.
\end{abstract} bios, Antillas.

Palabras clave: arquitectura militar, estudios bélicos, siglo XVIII, ataques anfi-

Abstract: The attacks on French settlements in the Caribbean during the Seven Years War can be considered an experiment prior to the one on Havana, probably the most significant victory of the conflict in the area. To reach this conclusion, this article analyzes the state of defensive constructions in Haiti, Guadeloupe, Martinique, Dominica and Saint Lucia. To this end, fortification projects deposited in different archives have been consulted, in addition to the architectural remains still preserved. Subsequently, the different British attacks developed between 1759 and 1762 are examined from primary sources, assessing the relevance of the fortifications in the result.

Keywords: military architecture, War studies, eighteenth century, amphibious attacks, Antilles.

$1 \quad$ Este trabajo forma parte de los resultados del Proyecto de Investigación I+D Ingenieros Militares en el Caribe y el Golfo de México Durante el Siglo XVIII. Diálogo Cultural, Circulación Transnacional y Conflictos Globales (HAR2015-63805-P), del que es Investigador Principal Alfredo f. Morales. 


\section{Introducción}

La contribución francesa a la fortificación de ciudades en el Caribe durante el siglo XVIII ha corrido una suerte dispar. Frente a los restos conservados en Guadalupe o Martinica, de difícil acceso en la actualidad, otros muchos han desaparecido, especialmente en la actual Haití, por lo que cobra especial importancia la información gráfica conservada en diferentes archivos. Ni siquiera en los casos conservados la historiografía más reciente ha mostrado un interés especial, con honrosas excepciones (Blanes, 2001, pp. 195-210; Verrand, 2004; Delpuech, 2007). En revisiones generales sobre las fortificaciones francesas de este siglo, las realizadas en el Caribe tienen una presencia testimonial (Langis, 2004; Lepage, 2010). Frente a estas, las levantadas más al norte han corrido mejor suerte (Chartrand, 2005). Todas ellas cuentan con especial actualidad a la luz de los recientes estudios que intentan analizar la Guerra de los Siete Años (1756-1763) como un conflicto de escala global, con un escenario particularmente rico en el ámbito caribeño (Baugh 2014). A la tradicional atención sobre los aspectos bélicos, se han ido sumando aproximaciones más interesadas en la labor de los ingenieros militares, lo que genera un campo de estudio más específico donde hacer conexiones trasnacionales con facilidad (Morales, 2016; Gunther, 2016; Luengo, 2017; Cruz, 2018; Gámez, 2018 y 2019).

Con esta situación, este artículo pretende demostrar que Francia contaba con estructuras defensivas suficientes en sus plazas más destacadas del Caribe para hacer frente a un ataque británico. Su pérdida no se debió tanto a problemas de actualización de las mismas con respecto a nuevas técnicas de asedio y a la artillería, sino a una deficiente gestión de los ya de por sí escasos pertrechos, en comparación con la dotación de los barcos británicos. Para demostrar esto se han tomado como casos de estudio las ciudades e islas atacadas por los británicos entre 1759 y 1762: Fort-de-France, Saint Pierre, Basseterre, Santa Lucia y Dominica. Se han localizado mapas de situación de sus defensas en esta fecha, conservados en diferentes archivos, y se han comparado con las memorias de los ataques publicadas por los británicos.

\section{Modelos de fortificaciones francesas en el Caribe a me- diados del siglo XVIII.}

Una rápida revisión de los proyectos franceses en sus posiciones en el Caribe muestra una importante apuesta en comparación con otras potencias europeas en la zona durante la misma fecha. Aunque 
la mayoría de los fuertes han sucumbido a los ataques de finales del siglo XVIII y principios del siglo XIX, las fuentes gráficas conservadas permiten valorar en detalle el grado de desarrollo y posterior eficacia de estas estructuras. Además, Francia optó por actualizar sus defensas en el Caribe de forma jerárquica. Así, Dominica no contaba con fortificaciones para estas fechas, Santa Lucía apenas presentaba algunas baterías, Basseterre (Guadalupe) un gran fuerte en plena actualización y Fort-de-France (Martinica) un sistema defensivo complejo puesto al día. Conocer bien el estado de cada una de las fortificaciones permitirá entender posteriormente el éxito y las dificultades de los ataques británicos como consecuencias y antecedentes de las campañas sobre plazas españolas como Cartagena (1741) o La Habana (1762).

\section{a. Saint-Domingue}

Con la ocupación del occidente de La Española a finales del siglo XVII, ratificado por el Tratado de Ryswick en 1697, la corona francesa se procuraba uno de los puntos estratégicos en el corazón del Caribe. Su dominio no sólo proveía a Francia de un territorio de enorme riqueza agrícola, sino que, flanqueando el Paso de los Vientos, se verificaba como un enclave militar de primer orden, desde el que controlar el acceso por el canal Viejo de Bahamas. A lo largo de la primera mitad del siglo XVIII se asiste a un rápido proceso de fortificación de todo su litoral que contrasta claramente con la indefensión a la que estaba expuesto durante el periodo español, situación que pervivirá en sus dominios orientales. El modelo de gestión territorial francés se fundamentará en la fundación de nuevos asentamientos en puntos estratégicos de la isla en donde se irán proyectando y construyendo diferentes fortificaciones. Se puede dividir el territorio en tres sectores principales coincidentes con sus litorales norte, este y sur, cuya importancia fluctuará a lo largo de la primera mitad del setecientos en función de las necesidades estratégicas que se materializarían en la fundación de diferentes capitales, lo que daría lugar a un marcado policentrismo que contribuiría decisivamente a la defensa de la colonia.

En las bahías de Jacmel y Les Cayes, en la banda sur de la isla, la Compagnie de Saint-Domingue radicaría sus sedes y almacenes (Pinon, 1999, pp. 112-113). La fundación de Saint Louis du Sud en la bahía homónima respondía a intereses estratégicos, tanto comerciales (Venegoni, 2013) -ubicada al centro de la gran península de Tiburón- como militares, por su resguardo y proximidad a Jamaica, en un punto desde el que los ingleses dirigieron su ataque en 1655. Por tal motivo, aquí se encontraba desde 1705 la primera fortificación de entidad de la isla (Moreau 
de Saint Mery, 1798, T. I, p. 625), conformada por un polígono irregular abaluartado adaptado a la morfología del islote en el que se levantó ${ }^{2}$ El ingeniero Renau será el autor del proyecto original que, según Moreu de Saint-Méry sería a la vez supervisado y corregido por Vauban (1798, T. I, p. 625). La vulnerabilidad del fuerte, necesitado de fuego de apoyo desde baterías de tierra, se mostraría evidente con motivo del ataque de Charles Knowles en 1748, lo que supone un antecedente de los innovadores sistemas de asedio británicos de décadas posteriores (Marley, 1998, p. 271).

Con sólo pequeños puntos destacados en Les Cayes, Jacmel, Torbech y Tiburón, esta fortificación sería la única de cierto porte en toda la banda meridional, enclave que sería pronto relegado por asentamientos más estratégicos en el norte de la isla. Aquí se fundó Port-de-Paix como sede de la gobernación de la colonia (Moreau de Saint Mery, 1798, T. I, p. 698$)^{3}$, donde se levantaría un sencillo fuerte que sería objeto de diferentes proyectos de reforma hasta la década de los años $40^{4}$. No obstante, acabaría perdiendo protagonismo frente a puertos mucho más defendibles como fueron los de Fort Dauphin y Cap Français. En este último se radicaría la capital del distrito norte tras su refundación en 1711 en una amplia y abierta bahía que a priori parecía suponer una clara desventaja defensiva, si bien su acceso quedaba condicionado por la existencia de numerosos bajos y escollos que obligaban a las embarcaciones a derrotar por un estrecho canal en cuyo tránsito se formarían varios proyectos de fortificación.

Desde 1736, la principal obra que cerraba la embocadura de este canal era Fort Picolet, una batería adaptada a la montaña escarpada que batía a dos niveles mediante sendas plataformas con troneras. Su gola se guardaba con un muro aspillerado, si bien su vulnerabilidad era insalvable al quedar totalmente dominado por las alturas circundantes 5 . De la misma manera estaba condicionada la batería de Saint Joseph que cerraba el paso por el segundo canal formado entre la costa y el bajo del Grande Mouton.

2 Biblioteca Nacional de Francia (BNF), Cartes et plans, GE SH 18 PF 152 DIV 4 P 17, Carte de l'Isle à Vache et de ses environs depuis la pointe d'Aquin jusqu'à la pointe de la Bacoüe, 1720.

3 BNF, Cartes et Plans, GE SH 18 PF 150 DIV 7 P 2 D, Plan du Port-de-Paix, Paul Corneau. 1686.

4 Archives Nationales d'Outre-Mer (ANOM), 15DFC439B, Plan du Port-de-Paix avec le projet, profil et élévation pour la réduction du fort et une batterie à faire à la Pointedes-Pères pour défendre la rade, Fean André du Coudreau, 1742.

5 ANOM, 15DFC334C, Plan, profil et elevation de la batterie sur la pointe Picolet, Louis-foseph La Lance, 1736. 
Desde aquí se abría el frente costero de la ciudad, que sería asimismo protegido por un cinturón de baterías, ideado en diferentes proyectos desde la década de los 40, aunque no sería llevado a la práctica, con carácter de campaña, hasta 1756 con el estallido de la guerra ${ }^{6}$.

Más inexpugnable parecía el puerto de Fort Dauphin, localizado en la profunda bahía de Bayaha en el límite oriental del territorio francés. El acceso quedaba controlado por un largo y estrecho canal en cuya bocana fue dispuesto Fort la Bouque, una extraña obra constituida por una batería curva flanqueada por orejones. Esta defensa hacía de avanzada del fuerte propiamente, formado por un reducto cuadrado en el centro de un foso comunicado con la referida plataforma mediante un puente levadizo (Blanes, 2001, pp. 201-202). En caso de salvar esta primera línea defensiva, el enemigo debía enfrentarse a los fuegos de las baterías de Saint Joseph y Saint Frederic, dispuestas en el tránsito del canal. Ya en el interior de la bahía, la ciudad quedaba protegida por Fort Dauphin, una obra poligonal abaluartada ceñida a la forma de una península que avanza sobre las aguas del fondeadero (Fig. 1) ${ }^{7}$.

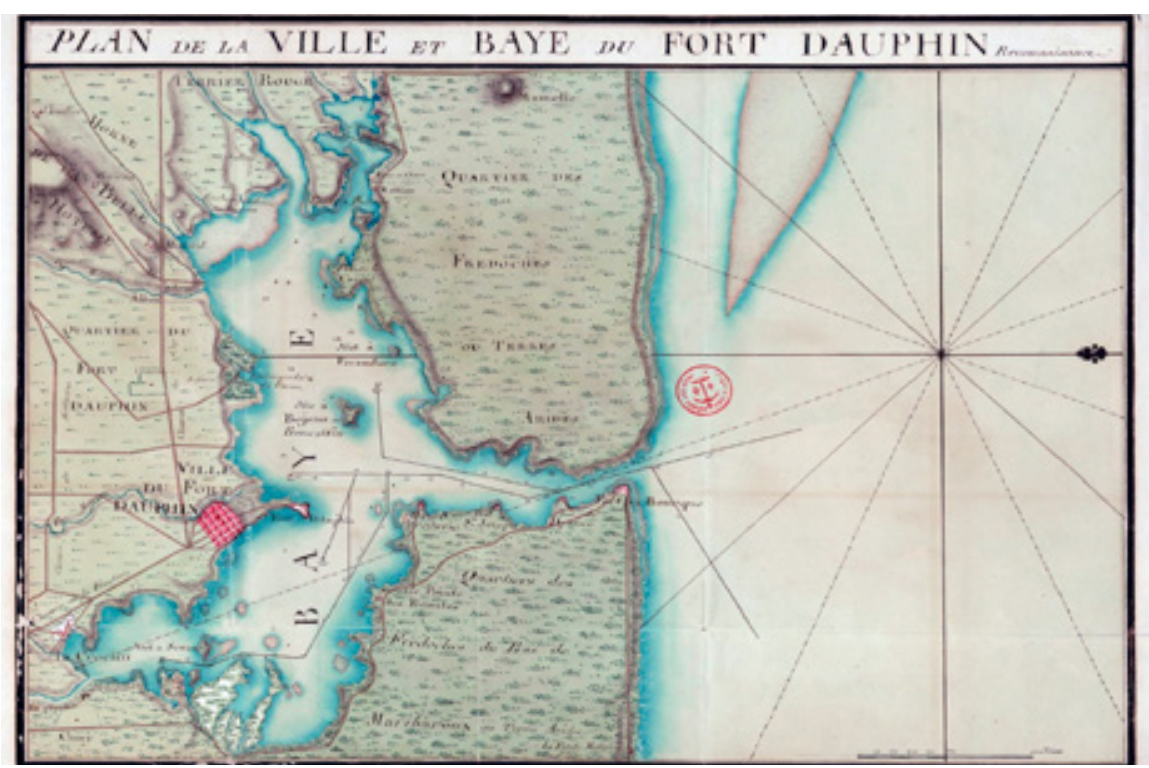

Fig.1 Anónimo, Plan de la Ville et Baye du Fort-Dauphin, c. 1764. FR_ANOM_15DF314B

6 ANOM, 15DFC362C, Plan de partie de la ville du Cap pour servir à faire voir les batteries en terre et les retranchements qui y ont été faits depuis le 20 octobre de l'année dernière qu'ils ont commencé, René Gabriel Rabié, 12 de octubre de 1756.

BNF, GE SH 18 PF 149 DIV 5P 6D, Plan de la baye, ville et fortifications du Fort-Dauphin, 1760;ANOM, 15DF314B, Plan de la Ville et Baye du Fort-Dauphin, c. 1764. 
El control del vasto litoral del golfo de Guanaba también precisaría de otras fundaciones. En la costa norte de la península de Tiburón se ubicó Petit Goâve como uno de los primeros asentamientos franceses ya desde 1663 (Moreau de Saint Mery, 1798, T. II, p. 537). Aquí se levantaría una pequeña batería que sería transformada mediante diferentes añadidos en un cuadrado abaluartado. Los ingenieros advirtieron pronto la exposición y vulnerabilidad del enclave, para lo que se formularon medidas como construir fuertes de apoyo o trasladar la población al otro extremo de la bahía, en un punto mucho más resguardado ${ }^{8}$. Estos proyectos serían contemplados para convertirla en capital central de la colonia, para lo cual sería barajada una nueva fundación en las llanuras del denominado Cul de Sac. Esta última voluntad prevalecería, dando lugar al nacimiento de Puerto Príncipe (Brown, 1837, Vol. 2, pp. 102103). La complicada navegación por sus aguas, surcadas de canales y escollos, procuraba un emplazamiento fácilmente defendible. Aunque desde su origen fue previsto su total amurallamiento, hacia 1753 se consideró suficiente controlar el islote llamado de Puerto Príncipe mediante el establecimiento de la batería de Saint Joseph ${ }^{9}$.

Hacia 1755, el mapa de poblaciones fortificadas de la isla se completaba con los asentamientos de Leogane, Saint Marc y Gonaïves, si bien, sus débiles baterías tenían un fin disuasorio frente a ataques piráticos, más que integrarse en el plan general de defensa de la colonia. Este último, se centraba más en diversificar sus centros, y establecer defensas fundamentadas solo en controlar pasos estratégicos frente a un ataque exclusivamente marítimo. Se abandonaría así el concepto francés de plaza fortificada de Vauban visto en Europa, impracticable en aquel medio, a pesar de haberse planteado en ocasiones su puesta en funcionamiento en algunos de los planes fundacionales citados.

\section{b. Guadalupe}

La fortificación de la isla de Guadalupe era compleja y se centraba en Basseterre en el sur de la isla, ya que Fort Louis seguía sin estar definitivamente construido. El caso de Basseterre era un asentamiento en una larga costa difícil de defender de un ataque naval, pero sin montes

8 ANOM, 15DFC716B, Plan du Petit Goave et du port de l'Acul proposé à fortifier, Herbert, 20 de diciembre de 1738.

9 ANOM, 15DFC610A, Plan du Port-au-Prince pour servir à la position des ouvrages les plus pressants pour la défense de sa rade, Mathias Henri Dumoulceau, 28 de agosto de 1753. 
suficientemente cercanos para suponer un riesgo. Quizás por esto, mantenía a mediados del siglo XVIII las estructuras tradicionales para la defensa. El Fuerte de Saint Charles, en la desembocadura del río Gallion era su obra principal, apoyada por cuatro baterías en la costa a un lado y otro de la ciudad. Ni siquiera en fuentes posteriores se han localizado referencias a baterías o reductos interiores ${ }^{10}$. Durante la primera mitad del siglo XVIII hubo mucha preocupación de los ingenieros allí destinados en reformar el Fuerte Saint Charles, alcanzando un diseño más moderno, con varios baluartes irregulares hacia tierra, dos revellines y una tenaza de acceso.

\section{c. Martinica}

La isla de Martinica era una de las posesiones más preciadas por la corona francesa en el Caribe, según lo demuestran sus fortificaciones (Verrand, 2014). Aunque a lo largo de la costa se ubicaron diferentes fuertes, como el de La Trinité en la parte oriental, o el de Saint Pierre en la occidental, el punto clave de su defensa era su capital Fort Royal (hoy Fort-de-France) (Kissoun, 2005). Esta ciudad se protegía con un doble sistema: un istmo fortificado lentamente desde principios del siglo XVIII que estaba ya terminado para este conflicto; y un alto número de baterías repartidas entre la costa y la cumbre de pequeñas lomas circundantes hacia el interior.

Según un plano anónimo del estado de las defensas del 19 de febrero de 1760 (Fig. 2), la artillería disponible en la ciudad se resumía en

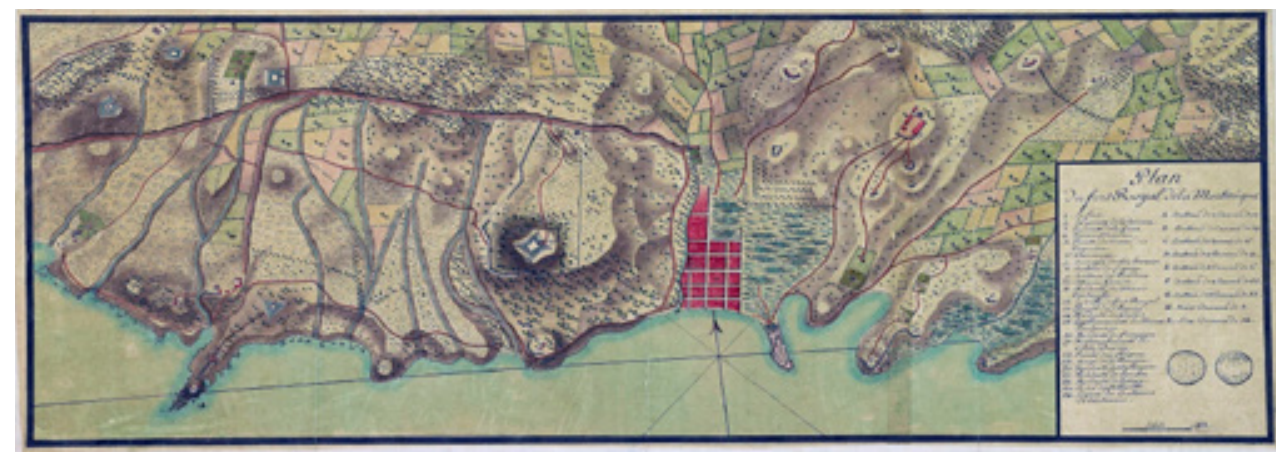

Fig.2. Anónimo. Plan du fort Royal de la Martinique. 19 de febrero de 1760.FR_ANOM_13DFC171C.

\footnotetext{
10 ANOM, 08DFC185A, Carte d'une partie de la Guadeloupe qui comprend la Basse-Terre et ses environs.
} 
diez cañones de 18 libras, cuatro de 12, dos de 8, tres de 6 y tres de $3^{11}$. Esta situación debía ser similar en un plano ligeramente posterior, del 15 de octubre de 1761, donde se representan con más detalle numerosas baterías en la costa: una en Pointe de la Case Navire, dos que protegían el desembarco junto a Mont Villeneuve, una en Point des Negres y dos más antes de llegar a la ciudad ${ }^{12}$. Ya hacia el interior de la bahía, una vez pasada la ciudad aparecían varias baterías en Point de la Cariere y Point a Grive. Estas baterías de apoyo suponían el sistema tradicional de protección ante ataques navales en el Caribe para la primera mitad del siglo XVIII, pero los franceses habían dado ya un paso más en la defensa, protegiendo también las colinas interiores. Así, en la parte occidental de la ciudad se había diseñado una línea de puestos avanzados sobre los montes en paralelo con el río Catherine. Justo detrás se encontraban varios reductos: Larcher, Calbacier, Villeneuve y Latapy. Con estos se controlaba una primera línea de ataque desde una serie de montes. Seguidamente se repetía el esquema con un nuevo monte y el consecuente río, con varios reductos llamados Tartanson. El frente de tierra de la ciudad, rodeada de colinas, estaba protegida con pequeñas baterías y reductos en el Monte Garnier y Cartouche.

Otra de las ciudades de la isla ya fortificada era Saint Pierre, una urbe en una rada al noroeste de la isla. Además del fuerte del siglo XVII que permanecía en la desembocadura del río, durante la primera mitad del siglo siguiente se construyeron diversas baterías en la línea de costa para reforzar una estructura ya arcaica. En cualquier caso, no se tiene constancia de que se levantara un fuerte abaluartado en un istmo cercano, como aparecería en representaciones inglesas de este momento (Fig. 3).

Con todo esto, resulta evidente que la fortificación propia de la primera mitad del siglo en la zona, caracterizada por grandes estructuras que defendían la entrada en el puerto de un ataque naval, había sido ampliamente superada. Ahora se temía un ataque anfibio con un gran protagonismo de la geografía circundante, aprovechando cualquier promontorio donde instalar artillería de un creciente alcance. Además de esto, parece claro que los trabajos para crear líneas de defensa eran mucho más profundos que la disponibilidad de artillería para dotarlos. El traslado entre puestos debía ser complejo por la falta de caminos y

11 ANOM, 13DFC171C, Plan du fort Royal de la Martinique, 19 de febrero de 1760.

12 ANOM, 13DFC177A, Plan des environs du Fort Royal de la Martinique, Henry de Rochemore, 15 de octubre de 1761. 


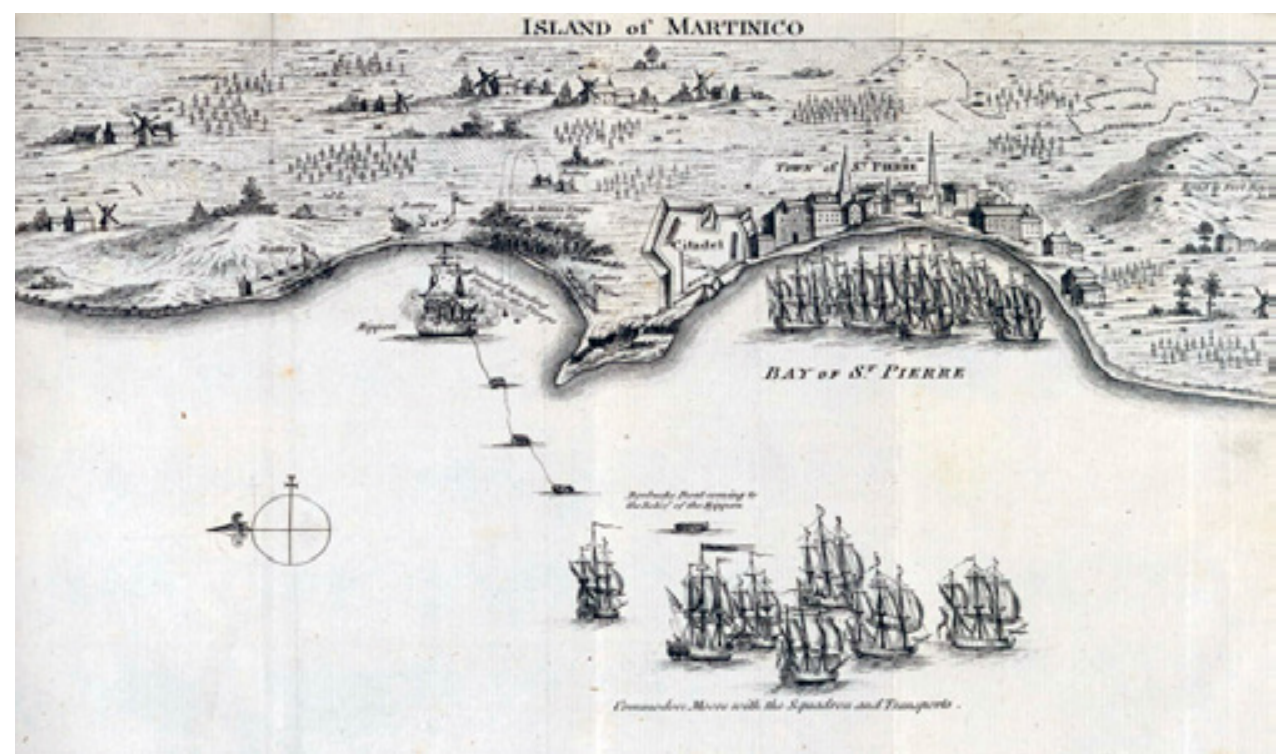

Fig.3. Island of Martinico. Birmingham: printed by fohn Baskerville; London: for G Steidel, at the Crown and Bible, Maddox-Street, Hanover-Square, 1762.

lo escarpado del terreno, por lo que se requería un plan de defensa que no llegaría hasta más adelante.

\section{d. Dominica y Santa Lucia}

El control de la isla de Santa Lucía, localizada a medio camino entre Martinica y Barbados, se evidenció en el siglo XVIII como una clara ventaja estratégica en tiempos de guerra. Es por ello que su estatus cambiará hasta en cuatro ocasiones a lo largo de la primera mitad de setecientos a merced de los acuerdos de paz firmados entre las coronas francesa y británica. Durante este periodo, la isla oscilará entre la soberanía francesa y la neutralidad, por lo que nunca se llegó a precisar un plan efectivo para su defensa, más allá de destacar baterías de campaña en los campamentos militares habilitados de forma transitoria ${ }^{13}$.

Al tiempo de la Guerra de los Siete Años, el control de la isla se localizaba en la bahía de Port du Carenage, en el litoral noroccidental insular. Allí se hicieron fuertes los ingleses con motivo de la ocupación

13 BNF, Cartes et Plans, GE SH 18 PF 157 DIV 9 P 4 D, Plan de Ste. Lucie, où sont marquées les differentes batteries, 1744. 
de Santa Lucía en 1723 (1755, T. I, p. 147), donde construyeron dos baterías que controlaban en alto el acceso a la bahía ${ }^{14}$. Bajo los mismos planteamientos defensivos, conocemos planes franceses para su total fortificación, que contemplaba la construcción de baterías y un tetrágono abaluartado dominando las alturas que flanqueaban la bocana de la bahía ${ }^{15}$. Sin embargo, a la conclusión de la guerra en 1763 tan sólo se encuentran restos de una batería en la punta de San Victor - presumiblemente desmontada tras la ocupación británica de 1762-y la levantada en 1723 en lo alto de la colina que flanquea el acceso ${ }^{16}$. Sólo se podría considerar este paraje como el único defendido de la isla, pues no resulta improbable que fuera usado el que se cita en varios mapas como ancient fort françois, frente al islote de Chocq, al norte de Port du Carenage.

Mayor indefensión presentaba hacia 1756 la isla de Dominica, la cual hasta finales del XVII tuvo estatus de isla neutral, donde se congregaron indios caribes. Su ocupación por parte de Francia, tuvo un carácter espontáneo durante el primer cuarto del siglo XVIII, por lo que no se localizan planes para su defensa. Tampoco tendría lugar después de que la administración colonial la tomara como propia y la dividiera territorialmente en 1727 (Boromé, 1972, p. 82; Honychurch, 1995, p. 50). Así permanecería aun después del tratado de Aix La Chapelle de 1748, cuando volvió a declararse neutral (Barberet y Magin, 1841, p. 176), si bien de nuevo, los asentamientos nunca llegaron a quedar bajo un control militar, al estar carente de valor estratégico para Francia al radicarse entre las islas de Guadalupe y Martinica.

En base a todos estos casos, se puede concluir que la propuesta francesa en el Caribe estaba basada en un trabajo profesional de ingenieros militares en permanente contacto con la metrópoli y que por tanto se encontraba razonablemente actualizado para las exigencias defensivas del momento, con las excepciones de Dominica y Santa Lucía motivadas por su situación extraordinaria.

14 BNF, Cartes et Plans, GE SH 18 PF 157 DIV 7 P 2, Plan des ances de la pointe du Chocq, et du port du Petit Carenage de l'isle Ste. Lucie avec les fortifications..., Pierre de Labarthe, 1723.

15 BNF, Cartes et Plans, GE SH 18 PF 157 DIV 9 P 2, Plan du port du Carenage ou Petit Cul de Sac de l'isle de Ste. Lucie, Fean-Baptiste Houel, 1742.

16 BNF, Cartes et Plans, GE SH 18 PF 157 DIV 6 P 2, Carte de l'isle de Sainte Lucie, facques-Nicolas Bellin y fean Baptiste Houel, 1763. 


\section{1759: El cambio de paradigma. Deficiencias del siste- ma ante los nuevos ataques británicos.}

El estudio de la historia de las fortificaciones suele dar pocas oportunidades para valorar la eficiencia de sus soluciones. En muchas menos ocasiones es posible valorar cómo un mismo grupo de militares es capaz de superar estos obstáculos en varias ciudades con perfiles fortificados similares en poco margen de tiempo, ya que esto permite evaluar el sistema defensivo eliminando otros factores. Esto se produce aquí, en lo que ha venido a llamarse la Campaña de las Islas Azucareras, entre 1759 y 1761 (Smelser, 1955). Aunque todas ellas terminaron sucumbiendo, resulta relevante diferenciar entre los ataques exclusivamente navales, adaptados a espacios abiertos al mar; de los planes anfibios, normalmente aplicados a ciudades con buena defensa naval, y con montes circundantes a menos de un kilómetro.

\section{e. Defensa de Fort-de-France (enero de 1759)}

El ataque sobre Martinica se inició en la mañana del 16 de enero sobre la batería de Point des Negres, que sólo resistió tres horas (Gardiner, 1762, p. 7). Desde aquí, los británicos optaron por desembarcar en Point de la Case Navire, más lejos de la ciudad, donde existía una batería. Aunque los franceses intentaron apoyarla desde Fort-deFrance, la batería de Negres dificultaba cualquier movimiento en este sentido. Las tropas desembarcaron en el punto previsto y se dirigieron a la capital. Al día siguiente, se posicionaron en uno de los montes cercanos, pero la defensa desde la ciudad hacía imposible el avance sin apoyo naval (1762, pp. 10-11). Tal situación llevó a la retirada y al embarco de tropa de nuevo en Point des Negres abortando la misión. La explicación de la fuente inglesa indica que no existían baterías desconocidas ni obstáculos imprevistos, por lo que no se entendía la retirada. En cambio, la relación no incluye muchas de las estructuras que por su ubicación en el interior debieron pasar desapercibidas de la inteligencia y causaron dudas sobre el potencial francés. Como conclusión, parece claro que los franceses contaban con un entramado defensivo exigente para el enemigo, aunque también de difícil uso que fue suficiente en esta ocasión.

De Fort-de-France, la campaña continuó en la cercana Saint Pierre. Aquí las crónicas confundieron incluso a los ilustradores, que ubicaron la ciudad en una bahía y no en una playa, además de confundir la fortificación (Fig. 3). La noticia del ataque británico ya se 
conocía, y aunque la ciudad estaba bien fortificada, apenas opuso resistencia (1762, pp. 14-16). El objetivo principal fue derrotar la ciudadela que probablemente no corresponda con el antiguo Fort Saint Pierre, ya muy desfasado, sino la recién terminada batería de Saint Louis y las baterías más pequeñas ubicadas en el extremo norte de la ciudad junto a los jesuitas ${ }^{17}$.

\section{f. Conquista de Basseterre (25 de enero de 1759)}

La campaña continuó con la conquista de Dominica, donde no encontró apenas resistencia en enero de 1759 (1762, p. 21). Algo más compleja fue la toma de Basseterre, aunque realizada en un modo más tradicional. Se inició destinando uno o varios barcos a cada una de las estructuras, siendo los tres mejor dotados los dedicados al fuerte Saint Charles (1762, p. 23) (Fig. 4). Poco tiempo duraría el intercambio,

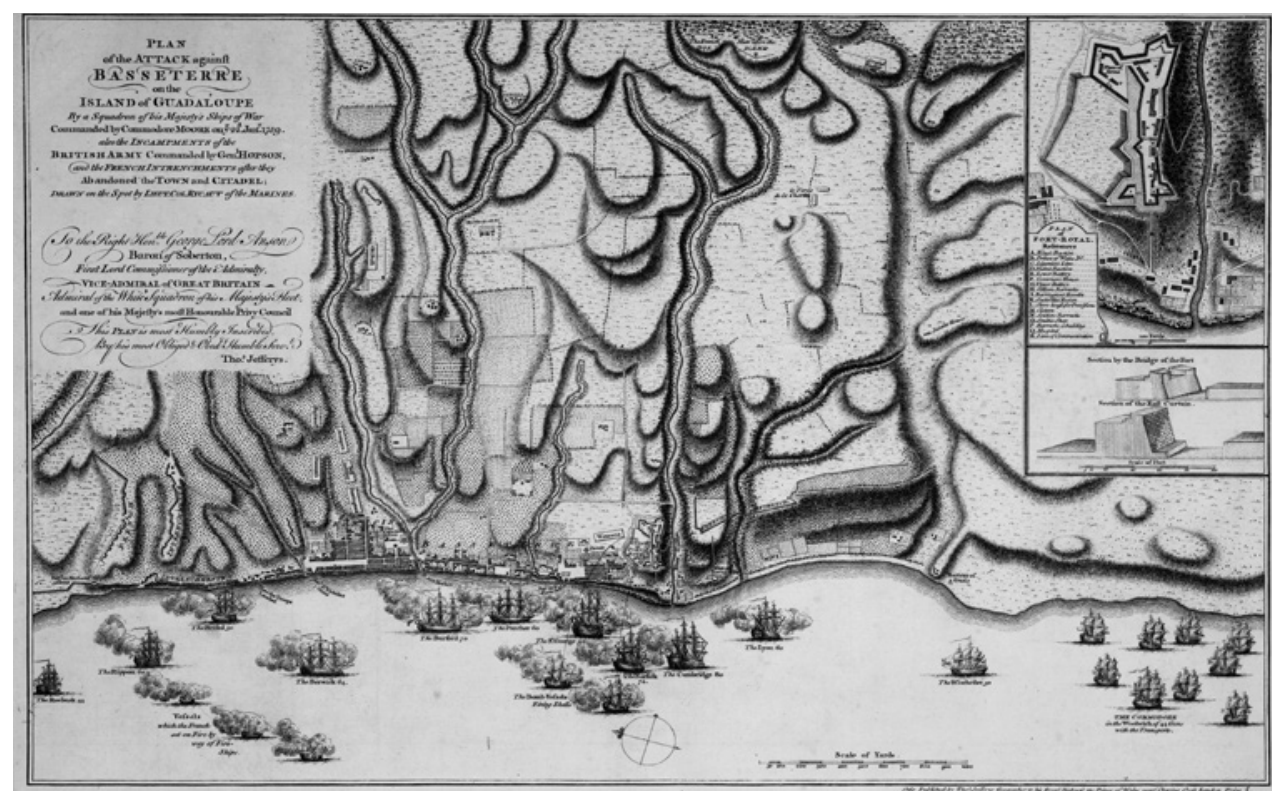

Fig.4. Fefferys, Thomas. Plan of the Attack against Basseterre on the Island of Guadaloupe by a Squadron of his Majesty's Ships of War Commanded by Commodore Moore on the 22nd Fanuary 1759.

Un ejemplo más fiel a lo que debió ser la fortificación lo presenta. BNF, Cartes et Plans, GE SH 18 PF 156 DIV 6 P6, Plan of the bay, town, fortifications and environs in the Island of Martinique, surveyed by order of G. B. Rodney in 1763, fohn Stott, 1763. 
huyendo los franceses antes incluso del desembarco, en el que no se entabló ningún enfrentamiento. Como conclusión es evidente que las tradicionales baterías no podían hacer frente con garantías al renovado potencial naval que ofrecían los británicos, siendo necesarias estructuras más complejas.

\section{g. Conquista de Fort-de-France (enero de 1762)}

Tres años hubo de esperar la corona británica para volver a intentar la toma de la capital de Martinica. La ciudad había procurado recuperarse y reforzar sus líneas. A pesar de esto, el sistema de ataque fue casi calcado al de 1759. El esfuerzo británico fue el de tomar el control del monte Mournier, donde se había construido un pequeño fuerte (Fig. 2). Como se observa en las pinturas contemporáneas de Dominic Serres, una vez consiguieron instalar la artillería allí, la ciudad quedaba a su disposición (Fig. 5). Las líneas defensivas francesas, mostradas en el sector

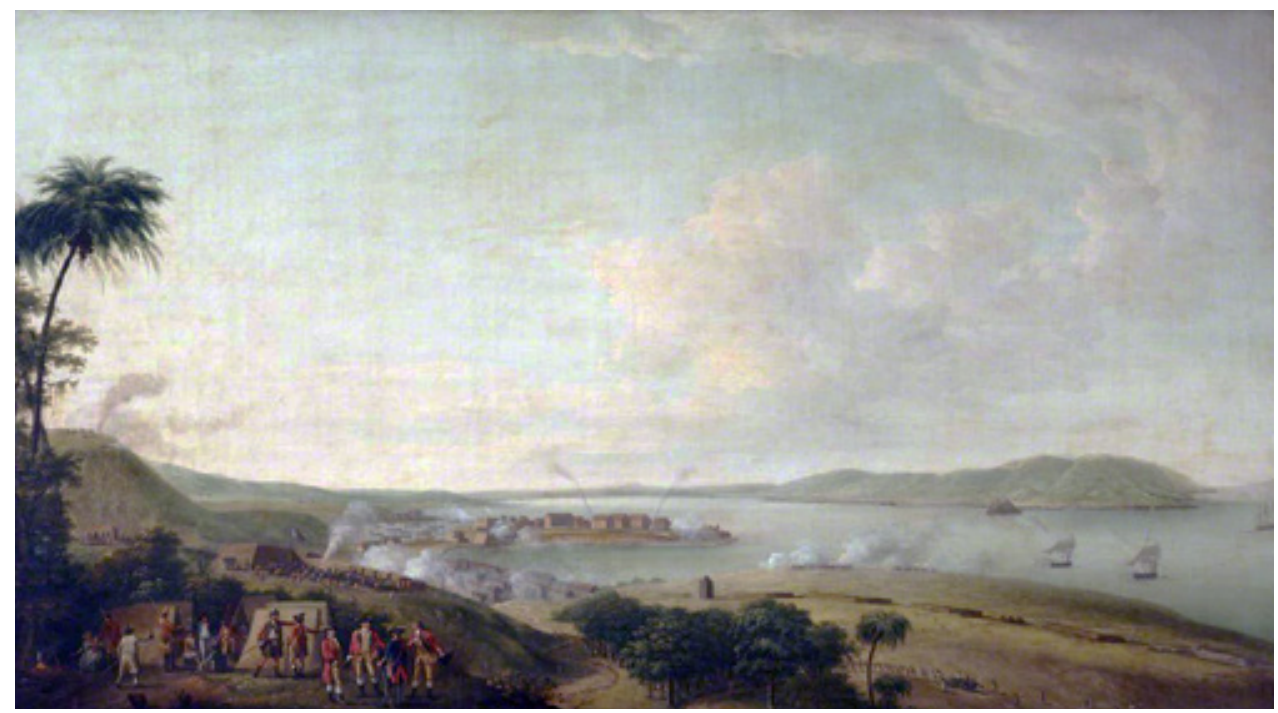

Fig.5. Dominic Serres. British Attack on the Citadel of Martinique, Fanuary 1762. National Trust, Anglesey Abbey, 515562.

derecho del cuadro, resultaban inútiles, al igual que el gran Fort Royal que daba nombre a la capital. En esta ocasión, el avance desde Case Navire fue más complejo, pero una semana después, la defensa francesa había quedado reducida a la ciudad. Una semana más tarde, Fort Royal se había rendido. Una vez obtenida una victoria significativa en 
Martinica, se continuó con otras islas más pequeñas como Santa Lucía en febrero de 1762 donde no se encontró resistencia desde tierra como muestra la vista de Serres (Fig. 6).

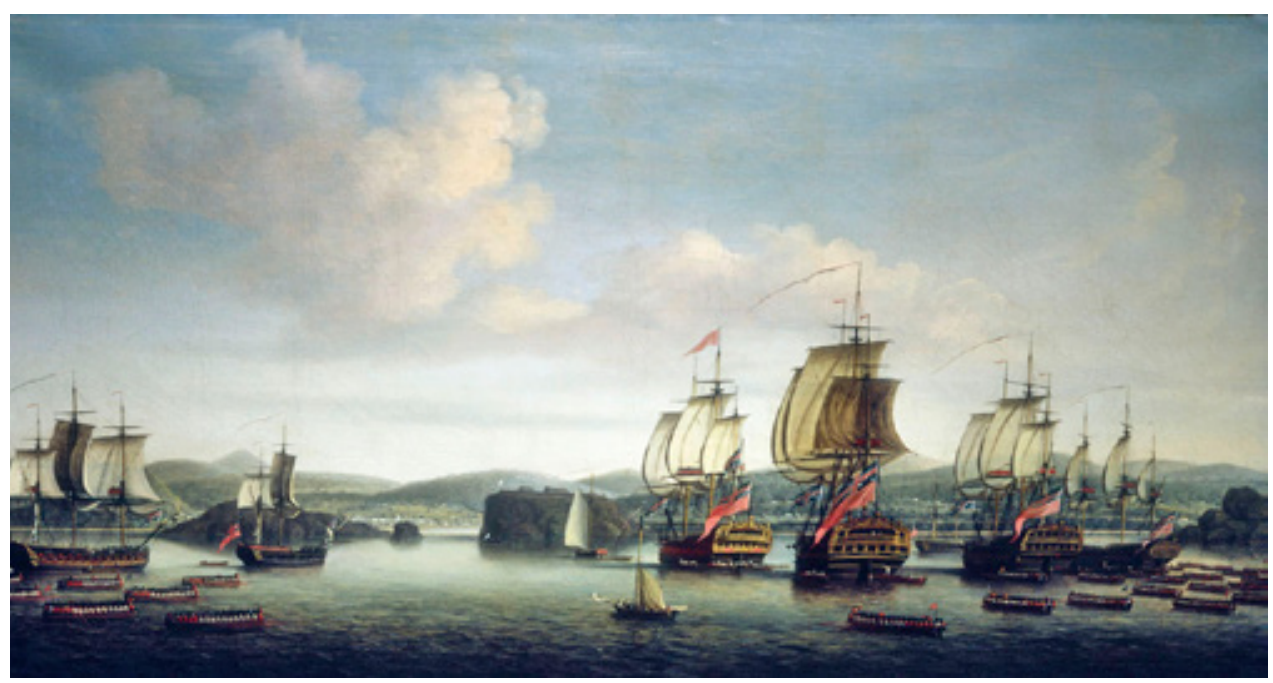

Fig.6. . Dominic Serres. The Capture of Saint Lucia, 26 February 1762. (C) National Maritime Museum, Greenwich, London. BHC0406

Estos ataques no sólo sirvieron para dar una importante ventaja británica en el conflicto sino para probar con éxito innovaciones tácticas que afectaban directamente a un diseño de fortificaciones que parecía inalterable desde los postulados de Vauban. Aunque los franceses habían intentado actualizarse en el Caribe antes de los ataques, sólo pudieron ralentizar puntualmente la efectividad de los nuevos procedimientos que de una u otra forma venían probándose desde el ataque sobre Saint Louis du Sud en 1748.

\section{Conclusiones}

Los ejemplos anteriores permiten alcanzar algunas conclusiones, a pesar de la complejidad del panorama bélico. En primer lugar, resulta evidente la apuesta francesa por la fortificación de sus plazas en el Caribe, especialmente las de Guadalupe, Martinica y la costa haitiana. En el caso de las dos primeras se hará uso de estructuras de gran formato y sistemas complejos, con un grado de actualización por lo general no visto en las defensas del archipiélago antillano. Por su parte, en la Saint Domingue francesa, el modelo defensivo sería otro. La multiplicación de puertos por la vasta extensión de su litoral no definía objetivos militares 
precisos cuya posesión comportara el domino de facto de todo el territorio dominicano, como por ejemplo sí ocurriría en Cuba con La Habana. Así, el despliegue defensivo francés aquí se fundamentaría en el control de los accesos a sus puertos mediante pequeños fuertes y baterías para hacer frente a ataques exclusivamente marítimos. A pesar del éxito británico en la campaña caribeña, el carácter disuasorio del modelo defensivo haitiano debe considerarse todo un acierto que nos pone sobre aviso de sistemas fortificados adaptados a las posibilidades y recursos de aquel territorio, de los que se conocen algunos precedentes en el caso español.

Con la excepción de Dominica y Santa Lucía, de menor trascendencia económica, se puede concluir que las fortificaciones pudieron haber sido suficientes para hacer frente a la campaña británica. El análisis de estos enfrentamientos insiste en que fue la superioridad en piezas de artillería y el torpe manejo de los fuertes lo que provocó la pérdida de las ciudades. En cambio, la actualización de las fortificaciones permitió ralentizar, y en algún caso impedir, las conquistas. Esto es especialmente interesante cuando se constata que los británicos estaban poniendo en marcha modelos de asedio novedosos. Frente al ataque naval más común en el Caribe del siglo XVII y primera mitad del siglo XVIII, la Guerra de los Siete Años se caracterizó por ataques multiflanco de carácter anfibio, en el que la orografía del terreno resultaba crucial. Si la pérdida de La Habana se debió a una deficiente defensa del monte de la Cabaña, esto se había comprobado previamente en los ataques a Martinica en 1759 y 1761. Esta innovación era especialmente trascendente para el ámbito caribeño, donde las ciudades más importantes del momento estaban bien protegidas ante un ataque naval, obviando estas opciones.

\section{Bibliografía}

(1755), Memoires des commissaires de Sa Majesté très-chretienne et de ceux de Sa Majesté brittannique: sur les possessions \& les droits respectifs des deux couronnes en Amerique, Ámsterdam-Leipzig: J. Schreuder \& P. Mortier le jeune.

BARBERET, Charles y MAGIN, Alfred (1841), Precis de Geographie Historique Universale, Paris: Chez Delamarche.

BAUGH, Daniel (2014), The Global Seven Years War. 1754-1763: Britain and France in a Great Power Contest, Londres-Nueva York: Routledge.

BLANES MARTÍN, Tamara (2001), Fortificaciones del Caribe, La Habana: Letras cubanas.

BOROMÉ, Joseph Alfred (1972), Aspects of Dominican History, 
Dominica: Government Print Division.

BROWN, Jonathan (1837), The history and present condition of St Domingo, Filadelfia: William Marshall and Co.

CHARTRAND, René (2005), French Fortresses in North America 1535-1763: Québec, Montréal, Louisbourg and New Orleans, Oxford-Nueva York: Osprey.

CRUZ, Pedro (2018), Silvestre Abarca Un ingeniero militar al servicio de la monarquía hispánica, Sevilla: Athenaica.

DELPUECH, André (2007), "Archéologie historique en Guadeloupe. Une nécessaire aproche du passé antillais", Les Nouvelles de l'archéologie, 108/109, no 101-112, pp. 101-112.

GÁMEZ CASADO, Manuel (2018), "Buscando al enemigo inglés. Expediciones de guardacostas españoles al golfo del Darién. 1767-1768", Anuario de Estudios Americanos, Vol. 75 (en prensa).

GÁMEZ CASADO, Manuel (2019), "Ingenieros militares en Nueva Granada durante el siglo XVIII. Movilidad, proyectos y expediciones", Revista de Indias. (en prensa).

GARDINER, Richard (1762), An Account of the Expedition to the West Indies, against Martinico, with the reduction of Guadelupe and other the Leeward Island. Birmingham: John Baskerville.

GUNTHER, Michael G. (2016) "British Military Engineering Efforts in the Hudson-Champlain Corridor in the Seven Years' War, and its aftermath", en Pedro Luengo y Gene Smith (eds), From Colonies to Countries in the North Caribbean: Military Engineers in the Development of Cities and Territories, Newcastle: Cambridge Scholar Publishing, pp. 1-22.

HONYCHURCH, Lennox (1995), The Dominica Story: A History of the Island, Londres: Macmillan, 1995.

KISSOUN, Bruno, (2005), "Fortifications des îles. Trois siècles d'architecture militaire en Guadeloupe: XVIIe-XIXe siècle", Bulletin Monumental, no 163-4, pp. 343-356.

LANGIS, Janis (2004), French Military Engineering from Vauban to the Revolution, Londres-Cambridge: The MIT Press.

LEPAGE, Jean-Denis, (2010), French Fortifications, 1715-1815: An Illustrated History, Londres: McFarland.

LUENGO, Pedro (2017), "Military Engineering in Eighteenth-Century Havana and Manila: The Experience of the Seven Years War", War in History, 24/1, pp. 4-27.

MARLEY, David (1998), Wars of the Americas: a chronology of armed conflict in the New World, 1492 to the Present, Oxford: ABC-CLIO.

MARSHALL, Smelser (1955), The Campaign for the Sugar Islands, 
1759. A Study of Amphibious Warfare, Chapel Hill: The University of North Carolina Press.

MORALES, Alfredo J (2016), "América y los ingenieros de Carlos III", en VV. AA., El Greco en su IV Centenario: Patrimonio hispánico y diálogo intercultural, Toledo: Universidad de Castilla la Mancha, pp. 67-78.

MOREAU DE SAINT MERY, M.L.E. (1798), Description topographique, physique, civile, politique et historique de la partie francaise de l'isle Saint-Domingue, T. I y II, Filadelfia: Chez l'auteur.

PINON, Pierre (1999), “Saint-Domingue: l'île à villes”, en Laurent Vidal y Émile d'Orgeix (eds), Les villes françaises du Nouveau Monde, París: Somogy éditions d'Art, pp. 108-119.

VENEGONI, Giovanni (2013), "De la Hermandad de la Costa a la Compañía Real de Saint Domingue: compañías comerciales, filibusteros y administración colonial en Santo Domingo, 1684-1720”, Boletín AFEHC, $\mathrm{n}^{\circ}$ 58. En: < http://www.afehc-historia-centroamericana.org/index. php?action=fi_aff\&id=3653> (Fecha de consulta: 31/01/2018)

VERRAND, Laurence (2004), "Fortifications militaires de Martinique, 1635-1845", Journal of Caribbean Archaeology, n 1, pp. 11-28. 